Theo Giannopoulos · Jeremy Chipchase

Anil Tailor · Simon Butler-Manuel

\title{
The use of harmonic shears (Ultracision) for laparoscopic lymphadenectomies in women with gynaecological malignancies
}

Received: 13 October 2004/ Accepted: 10 February 2005/Published online: 22 April 2005

(C) Springer-Verlag Berlin / Heidelberg 2005

\begin{abstract}
The purpose of this paper is to evaluate the safety and efficacy of the harmonic scalpel for laparoscopic pelvic and para-aortic lymphadenectomy in the management of gynaecologic cancers. A prospective study of 21 consecutive patients with gynaecologic cancer, including cervical (11), endometrial (3), ovarian (6) and tubal (1). Eleven patients had pelvic nodes retrieval only, two had para-aortic nodes retrieval only and eight had both. Eighteen patients had transperitoneal lymphadenectomies, one had extraperitoneal and two had both. Eleven patients had a hysterectomy or salpingooophorectomy in addition to the laparoscopic lymphadenectomy. In all cases, the harmonic scalpel was used as the only instrument to achieve haemostasis. Average nodal yields were 21.4 for bilateral pelvic lymphadenectomies. The mean estimated blood loss was $132.7 \mathrm{ml}$. There were no intraoperative complications. Laparoscopic pelvic and para-aortic lymphadenectomy using the harmonic scalpel appears to be both safe and efficient in the management of patients with gynaecologic cancers. Its main advantage is that it enables simultaneous and safe tissue dissection, haemostasis and cutting, avoiding the exchange of several instruments. It could become the first choice instrument for this procedure.
\end{abstract}

Keywords Lymphadenectomy - Laparoscopy · Harmonic shears

T. Giannopoulos $\cdot$ J. Chipchase $\cdot$ A. Tailor $\cdot$ S. Butler-Manuel Department of Gynaecology,

Royal Surrey County Hospital,

Guildford, United Kingdom

T. Giannopoulos $(\bowtie)$

Egerton Road, Guildford, Surrey,

GU2 7XX, United Kingdom

E-mail: tgiuk@yahoo.co.uk

\section{Introduction}

Laparoscopic lymphadenectomy is becoming increasingly common in the management of gynaecological malignancies, either in isolation or in combination with a vaginal procedure.

The avoidance of laparotomy leads to a shorter hospital stay, reduction in post-operative morbidity and earlier return to normal activities. In addition, the lymph node yield is comparable to the yield through laparotomy [1-5].

Laparoscopic transperitoneal lymphadenectomy in gynaecological oncology was first described by Dargent and Salvatin in 1989. The first laparoscopic extraperitoneal para-aortic lymphadenectomy was described by Vasilev and McGonigle in 1996 [6].

The majority of lymphadenectomies are still performed by laparotomy, as there is a steep learning curve for the laparoscopic procedure and also concerns of prolonged operating time and potential complications.

Laparoscopic lymphadenectomy was first described using bipolar diathermy and scissors, and this remains to be the standard technique. This requires the use of different instruments for coagulation, tissue dissection and cutting, together with frequent changes of instruments via the port sites.

In this paper, we describe our early experience with the use of 5-mm curved harmonic shears (Ultracision) for both transperitoneal and extraperitoneal lymphadenectomies.

\section{Methods}

We performed a prospective study of all women who underwent laparoscopic lymphadenectomies for various gynaecological malignancies.

Eligibility criteria required that the patients sign an informed consent prior to entry into the study. They were informed of potential complications related to the 
procedure (particularly, vascular injury and possibility of laparotomy).

Patients were judged ineligible for this study if they had contraindications to laparoscopy, such as bowel obstruction, ileus, peritonitis, diaphragmatic hernia, pregnancy or if they had another existing malignancy. Prior retroperitoneal surgery and prior pelvic or abdominal radiation were not considered as contraindications.

Fourteen patients were treated at the Royal Surrey Hospital Cancer Centre, United Kingdom, and the remaining seven were treated at two nearby independent hospitals.

Operative time was calculated from the first incision to completion of the surgery by using anaesthetic charts. Each woman was followed up until 8 weeks post-operatively, recording both the early and late complications. The total yield of lymph nodes was determined by histology.

\section{Operative technique}

Ultracision was used at the standard power levels of 5 (minimal coagulation, maximum cutting) and 2 (nearly maximum coagulation, minimal cutting).

For transperitoneal lymphadenectomy, four trocars were placed; a transumbilical, two lateral and a suprapubical. The umbilical trocars were $5 \mathrm{~mm}$ or $10 \mathrm{~mm}$ in size. The lateral trocars were $5 \mathrm{~mm}$. The suprapubical was $12 \mathrm{~mm}$ and was used for tissue retrieval, and also to provide retraction of tissues (principally, the sigmoid colon). A Verres needle was inserted transumbilically, after performing a Palmers test. The initial intraabdominal pressure (achieved with the Verres needle) was $25 \mathrm{~mm} \mathrm{Hg}$, which was then lowered to $18 \mathrm{~mm} \mathrm{Hg}$ after the insertion of the primary trocar. The rest of the procedure was performed as described by Querleu et al. [7].

The extra peritoneal lymphadenectomy was performed in the supine position, as described by Dargent et al. [8].

For all cases, drains were not routinely used but surgicell was left in the obturator fossa or the retroperitoneal space to improve haemostasis (as it is our normal practice for open lymphadenectomies). All patients had one dose of intraoperative antibiotics (metronidazole and cefuroxime). In all cases, the nodes were removed with an endopouch.

\section{Results}

Twenty-one patients underwent laparoscopic lymph node dissections as part of their treatment for a variety of gynaecologic malignancies from October 2002 to August 2004. The study group included 11 patients with cervical cancer, three with endometrial, six with ovarian and one with fallopian tubes cancer.
The mean age was 43.4 years (range 25-81). Eleven patients had a hysterectomy or salpingo-oophorectomy in addition to the laparoscopic lymphadenectomy, including six radical vaginal hysterectomies and five laparoscopically assisted vaginal hysterectomies. All of the radical vaginal hysterectomies patients had early-stage cervical cancer. The remaining ten patients had laparoscopic lymphadenectomies without an accompanying hysterectomy (Table 1). Eleven patients had pelvic nodes retrieval only, two had para-aortic nodes retrieval only and eight had both. All cases were done either by or under the direct supervision of a subspecialist gynaecological oncologist (SBM).

\section{Lymph node yield}

All of the 21 patients in this study had nodal tissue retrieved, as confirmed by histological examination. The mean number of harvested nodes in 11 patients who had bilateral pelvic lymphadenectomies was 21.4 (range1144).

The remaining ten women had lymph node biopsies, as directed by imaging and, therefore, the number of nodes was within a range of 1-6.

Eighteen patients had transperitoneal lymphadenectomies, one had extraperitoneal and two had both.

\section{Operative time and estimated blood loss}

The mean lymphadenectomy operating time was $110.2 \mathrm{~min}$ (range 60-21 min). The mean estimated blood loss was $132.7 \mathrm{ml}$, with a range of $50-400 \mathrm{ml}$. In all cases, Ultracision was used as the only instrument to achieve haemostasis. Figure 1 shows the operating time for consecutive cases of patients that had bilateral pelvic lymphadenectomies.

\section{Intraoperative and postoperative complications}

No major complications occurred. Also, there have been no cases of trocar site recurrences.

Length of stay

All of the ten patients who had lymphadenectomies only undertook an overnight stay.

Table 1 Characteristics of patients having laparoscopic procedures only

\begin{tabular}{llllll}
\hline & $\begin{array}{l}\text { Stage } \\
\text { IA }\end{array}$ & $\begin{array}{l}\text { Stage } \\
\text { IIB }\end{array}$ & $\begin{array}{l}\text { Stage } \\
\text { IIIA }\end{array}$ & $\begin{array}{l}\text { Stage } \\
\text { IIIB }\end{array}$ & $\begin{array}{l}\text { Stage } \\
\text { IIIC }\end{array}$ \\
\hline $\begin{array}{l}\text { Cervical cancer } \\
\begin{array}{l}\text { Ovarian/tubal } \\
\text { cancer }\end{array}\end{array}$ & 1 & 3 & 1 & 1 & \\
\hline
\end{tabular}




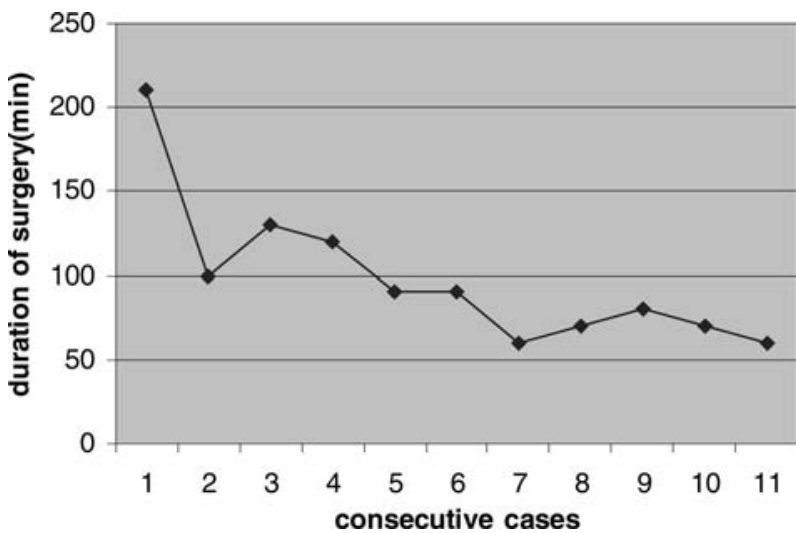

Fig. 1 Operative time in consecutive cases of bilateral pelvic lymphadenectomies

The hospital stay for the rest ranged from 2 days to 8 days (mean 4.1 days).

\section{Discussion}

We found that Ultracision worked very well as a multifunctional instrument, and by using, it we minimised the need for instrument exchanges as it permitted multiple functions.

In addition to its function as a knife and as scissors, Ultracision was also used without power as a grasper and as a dissector. It coagulated, dissected and cut both supported and unsupported tissue, vessels and highly vascular structures without instrument exchanges. The effect of coagulation was controlled by activation duration and power level to protect against tissue damage.

The system of the harmonic shears consists of a generator, a reusable handpiece and shears. It functions by destroying cellular tissue by desiccation and denaturation and achieves this effect by mechanical oscillation at a frequency of $55,000 \mathrm{~Hz}$. This high frequency prevents the shears from adhering to the tissue surface. Known advantages of mechanical destruction include less thermal damage to the surrounding tissue and the absence of after-burning smoke.

Compared to electrosurgery and laser surgery, Ultracision appears to provide us with potential advantages. It is probably safer than bipolar diathermy, as no electrical energy passes to or through the patient and, also, there is no risk of electric shock from the harmonic scalpel line of instruments $[9,10]$. Compared to monopolar diathermy, where thermal injury can occur within a second of use, the harmonic scalpel offers a much more controllable source of energy [11]. The use of Ultracision probably reduces the risk of abdominal wall vascular trauma, as there is no need for frequent changes of instruments.

Also, the visual field is clearer, as there is minimal smoke. This is because it cuts and coagulates tissues at lower temperatures (less than $100^{\circ} \mathrm{C}$ ). The lateral thermal spread also appears less, compared to what is seen with electrosurgery [12-14]. On the contrary, it probably coagulates slower than bipolar diathermy; however, it saves operative time as there is no need for changes of instruments.

Compared with the laser, it offers the advantage of easier set-up and fewer precautions for the theatre staff.

The cost of a disposable set for the harmonic scalpel is approximately $£ 300(\sim € 440)$. This is much higher than that of the mostly re-usable set for surgical diathermy. However, savings are made by minimising the use of other instruments that are needed when electrosurgery is being used (i.e. scissors).

In our series, all the targeted lymph nodes were harvested and there was no need to proceed to laparotomy to complete the procedure. Our operative time and intraoperative blood losses are comparable to what is published from other researchers who used either electrocautery or Ultracision technology [15-18]. As expected, the operative time decreased as we became more familiar with the use of the instrument (Fig. 1). In none of our cases did we have to resort to bipolar diathermy to secure haemostasis.

The management of all our patients who had laparoscopic lymphadenectomy was influenced by the histological assessment of nodes. This information could not have been obtained by currently available crosssectional imaging modalities, since they are associated with relatively poor sensitivity and specificity [19-22].

To our knowledge, there is very limited published data as regards to the use of Ultracision for laparoscopic lymphadenectomies [23, 24]. Our data supports the evidence from other researchers and compares favourably with the results of other studies that used electrosurgery for this procedure.

Overall, it appears that lymphadenectomy with Ultracision is a technically feasible procedure with an acceptable safety profile, satisfactory nodal yield and short hospital stay. We appreciate that the number of patients in our study is small, but we are encouraged by these early results and continue to recruit patients in our study.

As there are increasing indications for lymphadenectomy in gynaecological oncology, it is likely that Ultracision will evolve as an ideal instrument for this procedure. By being multifunctional and ergonomic, it will probably shorten the operative time and make a difficult procedure easier, safer and faster. Of course, there is a learning curve, like with all new techniques, but, overall, we found that it is quite user-friendly and a very reliable piece of equipment. As expected, the operative time decreased as more experience with this instrument was acquired (Fig. 1).

In our opinion, Ultracision could become a first choice instrument for laparoscopic lymphadenectomy, as it enables simultaneous safe tissue dissection, haemostasis and cutting. We think that its favourable features will encourage more surgeons to perform laparoscopic lymphadenectomies. 


\section{References}

1. Chu KK, Chang SD, Chan FT, Soon YK (1997) Laparoscopic surgical staging in cervical cancer-preliminary experience among Chinese. Gynecol Oncol 64:49-53

2. Possover M, Krause N, Plaul K, Kiihne-Heid R, Schneider A (1998) Laparoscopic para-aortic and pelvic lymphadenectomy: experience of 150 patients and review of the literature. Gynecol Oncol 71:19-28

3. Fowler J, Carter J, Carlson JW, Maslonkowski R, Byers LJ, Carson LF, Twiggs LB (1993) Lymph node yield from laparoscopic lymphadenectomy in cervical cancer: a comparative study. Gynecol Oncol 51:187-192

4. Querleu D, Dargent D, Ansquer Y, Leblanc E, Narducci F (2000) Extraperitoneal endosurgical aortic and common iliac dissection in the staging of bulky or advanced cervical carcinomas. Cancer 88(8):1883-1891

5. Schlaerth JB, Spirtos NM, Carson LF, Boike G, Adamec T, Stonebraker B (2002) Laparoscopic retroperitoneal lymphadenectomy followed by immediate laparotomy in women with cervical cancer: a gynecologic oncology group study. Gynecol Oncol 85(1):81-88

6. Vasilev SA, McGonigle KF (1996) Extraperitoneal laparoscopic paraaortic lymph node dissection. Gynecol Oncol 61:315-320

7. Querleu D, Leblanc E, Castelain B (1991) Laparoscopic pelvic lymphadenectomy in the staging of early carcinoma of the cervix. Am J Obstet Gynecol 164:579-581

8. Dargent D (1999) Retroperitoneal approach for lymph-node sampling and dissection. In: Querleu D, Childers JM, Dargent D (eds) Laparoscopic, surgery in gynecological oncology, 1st edn. Blackwell, London, pp 34-40

9. Ott DE, Moss E, Martinez K (1998) Aerosol exposure from an ultrasonically activated device. J Am Assoc Gyn Lapar 5(1):2932

10. Beebe DS, Swica H, Carlson N, Palahnjuk RJ, Goodale RL (1993) High levels of carbon monoxide are produced by electrocautery of tissue during laparoscopic cholecystectomy. Anesth Analg 77:338-341

11. Esper E, Russel TE, Coy B, Duke BE 3rd, Max MH, Coil JA (1994) Transperitoneal absorption of thermocautery-induced carbon monoxide formation during laparoscopic cholecystectomy. Surg Laparosc Endosc 4:333-335
12. Ammaral JF (199) The experimental development of an ultrasonically activated scalpel for laparoscope use. Surg Laparoendose 4:92-99

13. Schwarz RO (1994) Total laparoscopic hysterectomy with the harmonic scalpel. J Gynecol Surg 10:33-34

14. Robbins ML, Ferland RJ (1995) Laparoscopic-assisted vaginal hysterectomy using the laparoscopic coagulating shears. J Am Assoc Gynecol Laparosc 2:339-343

15. Schlaerth J, Spirtos N, Carson L, Boike G, Adamec T, Stonebraker B (2002) Laparoscopic retroperitoneal lymphadenectomy followed by immediate laparotomy in women with cervical cancer. Gynecol Oncol 85:81-88

16. Kadar N (1995) Laparoscopic pelvic and aortic lymphadenectomy. Baillieres Clin Obstet Gynaecol 9(4):651-673

17. Mehra G, Weekes AR, Jacobs IJ, Visvanathan D, Menon U, Jeyarajah AR (2004) Laparoscopic extraperitoneal paraaortic lymphadenectomy: a study of its applications in gynecological malignancies. Gynecol Oncol 93(1):189-193

18. Dottino PR, Tobias DH, Beddoe A, Golden AL, Cohen CJ (1999) Laparoscopic lymphadenectomy for gynecologic malignancies. Gynecol Oncol 73:383-388

19. Narayan K, Hick RJ, Jobling T, Bernshaw D, Mckenzie AF (2001) A comparison of MRI and PET scanning in surgically staged loco-regionally advanced cervical cancer: potential impact on treatment. Int J Gynecol Cancer 11:263-271

20. Greco A, Mason P, Leung AWL, McIndoe GAJ, Anderson MC (1989) Staging of carcinoma of the uterine cervix: MRIsurgical correlation. Clin Radiol 40:401-405

21. Hawnaur JM, Johnson RJ, Buckley CH, Tindall V, Isherwood I (1994) Staging, volume estimation and assessment of nodal status in carcinoma of the cervix: comparison of magnetic resonance imaging with surgical findings. Clin Radiol 49:443452

22. Kim SH, Kim SC, Choi BI, Han MC (1994) Uterine cervical carcinoma: evaluation of pelvic lymph node metastasis with MR imaging. Radiol 190:807-811

23. Nezhat F, Miranda C, Rahaman J, Gretz H, Cohen C (2002) Laparoscopic lymphadenectomy for gynecologic cancer using the harmonic scalpel: a feasibility study. Obstet Gynecol 99(4):S34

24. Holub Z, Jabor A, Kliment L, Voracek J, Lukac J, Barany B (2001) Laparoscopic staging of endometrial cancer using laparosonic instruments: comparison with electrosurgery. Euro J Obstet Gynecol Reprod Biol 100:81-86 